\title{
Is the commissural nucleus of the solitary tract essential for the maintenance of renovascular hypertension? A putative role for the carotid bodies
}

\author{
Valdir A. Braga ${ }^{1,2}$ \\ Received: 5 January 2019 / Revised: 11 January 2019 / Accepted: 11 January 2019 / Published online: 21 February 2019 \\ (c) The Japanese Society of Hypertension 2019
}

Renovascular hypertension is a type of secondary hypertension that corresponds to $5-10 \%$ of hypertensive patients and is considered a severe but treatable form of the disease [1]. Regarding its pathophysiology, the classical studies performed by Goldblatt et al. [2] in the 1930s demonstrated that a reduction in kidney perfusion led to sustained elevation of arterial pressure. A few decades later, elevation of the circulating angiotensin II (ANG II) level was suggested as the main mechanism underlying the increase in blood pressure seen in renovascular hypertension [3]. With the advent of the angiotensin-converting enzyme inhibitor captopril, the concept that renovascular hypertension is a form of ANG II-dependent hypertension has been clarified [3].

Several studies have investigated how an increase in the circulating ANG II level leads to hypertension. Considering that circulating ANG II peptides are too large to cross the blood-brain barrier (BBB), one theory suggests that ANG II exerts its effects by acting on circumventricular organs, such as the subfornical organ (SFO), area postrema (AP), and organum vasculosum of the lamina terminalis [4]. One of the major pathways by which circulating ANG II mediates increases in sympathetic activity resulting in arterial hypertension is the SFO-paraventricular nucleus of the hypothalamus (PVN)-rostral ventrolateral medulla (RVLM) axis. According to this theory, blood-borne ANG II acts via circumventricular organs and leads to de novo synthesis of ANG II within brain areas involved in cardiovascular

Valdir A. Braga

valdir@cbiotec.ufpb.br

1 Department of Physiology and Pharmacology, Karolinska Institutet, Stockholm, Sweden

2 Biotechnology Center, Federal University of Paraiba, Joao PessoaPB, Brazil control, such as the PVN and RVLM [4]. This increase in ANG II signaling within the brain involves NADPH oxidase activation, reactive oxygen species formation, endoplasmic reticulum stress, inflammation, and nuclear transcription factor activation, which may alter neuronal firing, especially in baroreflex-sensitive presympathetic neurons in the RVLM [5]. More recently, Biancardi et al. [6] suggested that during hypertension, circulating ANG II could gain access to neurons and glial cells within hypothalamic (i.e., PVN) and brainstem (i.e., nucleus of the solitary tract (NTS) and RVLM) regions due to disruption of BBB permeability, which seemed to involve activation of $\mathrm{AT}_{1}$ receptors. Despite compelling evidence, there is still no consensus on how circulating ANG II affects the brain areas involved in cardiovascular control. The mechanism probably includes a combination of the two theories mentioned above.

One of the key brain regions involved in cardiovascular regulation is the NTS. The NTS, which is located in the dorsomedial portion of the medulla, is the first synaptic station for visceral afferents and integrates a wide variety of reflexes controlling cardiovascular function, respiration, and gastrointestinal motility [7]. Anatomically, the NTS can be subdivided into rostral, intermediate, and caudal regions, each of which includes different subnuclei defined based on their positions relative to the solitary tract. One important NTS subnucleus is the commissural NTS (cNTS), which receives converging inputs involved in processing of the peripheral chemoreflex via oxygen sensors located in the bifurcation of the carotid arteries (i.e., the so-called carotid bodies) [8]. Recently, the carotid bodies and the integration and processing of their afferents into the cNTS in the context of arterial hypertension have gained attention. For instance, Pijacka et al. [9] demonstrated that overactivity of the peripheral chemoreceptors via purinergic P2X3 receptors in the carotid bodies generated aberrant signaling to the cNTS that contributed to hypertension. In addition, clinical studies showed that unilateral resection of the carotid body 


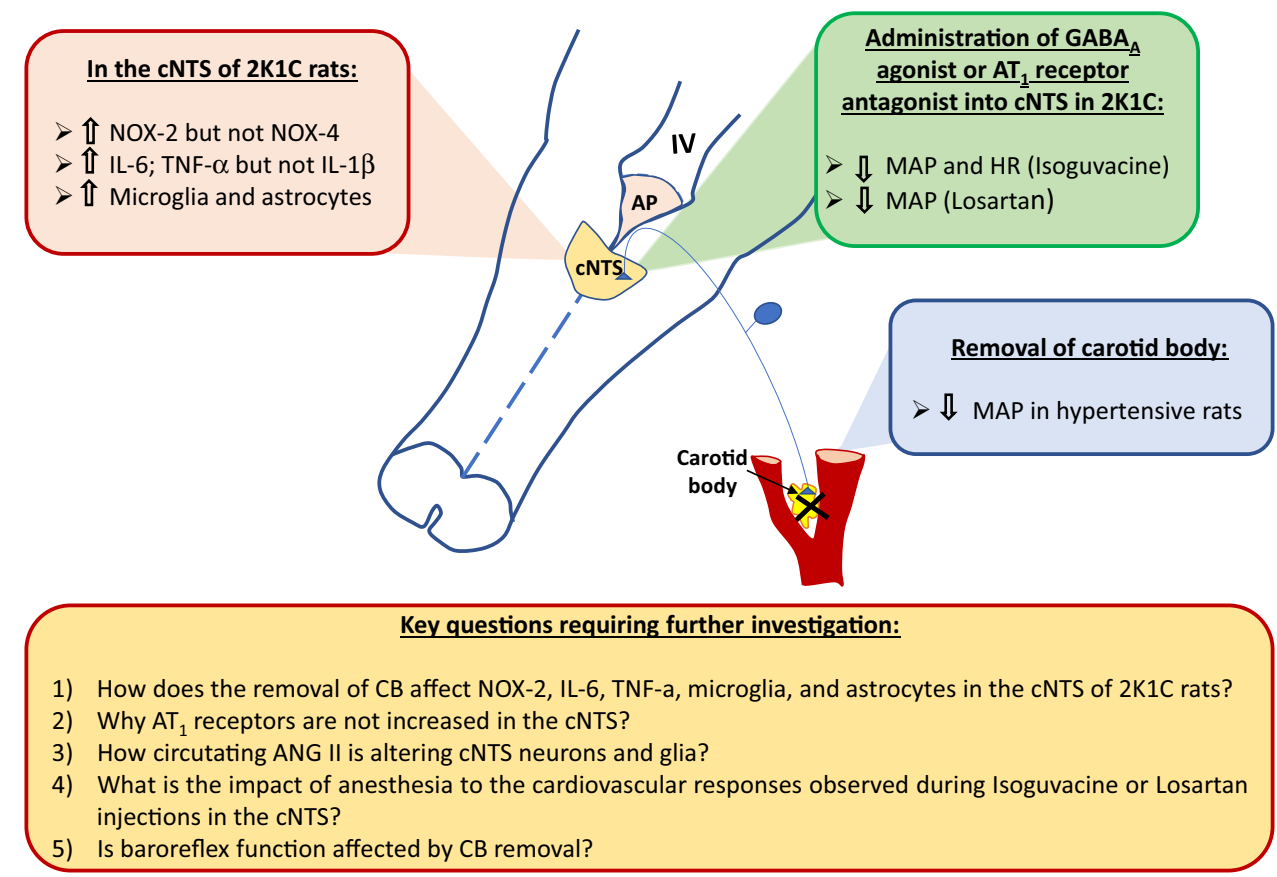

Fig. 1 Schematic drawing summarizing the findings of Melo et al. [12] and highlighting some questions that should be addressed in future investigations. The authors demonstrated that NOX-2, IL-6, TNF- $\alpha$, microglia, and astrocytes were elevated in the cNTS of the 2-kidney-1-clip (2K1C) experimental model of renovascular hypertension. Furthermore, activation of $\mathrm{GABA}_{\mathrm{A}}$ receptors by isoguvacine or inhibition of $\mathrm{AT}_{1}$ receptors with losartan in the cNTS attenuated hypertension. Lastly, the authors documented that the removal of carotid bodies 3 weeks after renal artery clipping blunted development of renovascular hypertension. NOX-2 nicotinamide adenine dinucleotide phosphate oxidase isoform 2, IL-6 interleukin 6, TNF- $\alpha$ tumor necrosis factor-alpha, $\mathrm{GABA}_{\mathrm{A}} \gamma$-aminobutyric acid type $\mathrm{A}$ receptor, $\mathrm{AT}_{1}$ angiotensin type 1 receptor, IV fourth ventricle of the brain;,AP area postrema, cNTS commissural nucleus of the solitary tract, MAP mean arterial pressure, HR heart rate, CBs carotid bodies

in patients with resistant hypertension was beneficial [10]. Although the carotid bodies evidently play a role in some forms of hypertension [11], their roles in the integration and processing of information at the cNTS level in ANG IIdependent hypertension have not been investigated.

In this issue of Hypertension Research, Melo et al. [12] make an important contribution to understanding how carotid bodies may influence neurotransmission at the cNTS to maintain renovascular hypertension. Using a combination of refined in vitro and in vivo approaches (Fig. 1), the authors demonstrated that activation of GABAergic receptors or inhibition of $\mathrm{AT}_{1}$ receptors in the cNTS blunted hypertension in 2-kidney-1-clip (2K1C) rats. In addition, markers of oxidative stress (NOX-2) and inflammation (tumor necrosis factor-alpha (TNF- $\alpha$ ) and interleukin (IL)6) were elevated together with increased microglia and astrocyte numbers in the cNTS. Lastly, removal of the carotid bodies after 3 weeks of renal artery clipping compromised the progression of renovascular hypertension. Therefore, the authors suggested that the carotid bodies tonically activated neurons in the cNTS, which was essential for maintenance of renovascular hypertension. Furthermore, they concluded that ANG II played an important role in the neural processing of renovascular hypertension by evoking microgliosis and astrogliosis within the cNTS, probably by enhancing oxidative stress and inflammation.

Although the novelty of this study is unquestionable, some key questions remain open and need further investigation (Fig. 1). First, how does the removal of carotid bodies affect NOX-2, IL-6, TNF- $\alpha$, microglia, and astrocytes in the cNTS of $2 \mathrm{~K} 1 \mathrm{C}$ rats? Although the authors demonstrate that both phenomena take place in separate experiments, measurements of oxidative stress (i.e., superoxide, NOX-2, SOD1, SOD2, and catalase) and inflammatory markers (i.e., IL-6, TNF- $\alpha$, and IL-10), microglia and astrocytes should be performed in hypertensive rats after carotid body removal to yield evidence of interactions among those findings rather than parallel observations. Second, why did $\mathrm{AT}_{1}$ receptor mRNA expression not change in the cNTS of $2 \mathrm{~K} 1 \mathrm{C}$ rats? There is compelling evidence that increasing ANG II in other brain regions involved in cardiovascular control, such as the RVLM, increases $\mathrm{AT}_{1}$ receptor expression [13]. Considering that NOX-2 expression is increased in the cNTS during hypertension, we can reasonably suspect overactive angiotensinergic signaling in that region. Third, how does circulating ANG II affect neurons and glia in the cNTS? Given the proximity with the AP, one question that needs to be addressed is whether ANG II is acting at the cNTS via 
direct angiotensinergic projections from the AP, which is a circumventricular organ, leading to de novo ANG II synthesis in the cNTS or whether circulating ANG II is causing a breakdown in the BBB, thereby allowing direct access of the neurons and glia of the cNTS to circulating ANG II. Fourth, what is the impact of anesthesia on the cardiovascular responses observed during isoguvacine or losartan injections in the cNTS? Urethane anesthesia is well accepted to depress neurotransmission in the NTS. Therefore, investigating GABAergic and angiotensinergic mechanisms within the cNTS during renovascular hypertension in a context free of anesthesia is important. Lastly, is the baroreflex function affected by carotid body removal? The baroreflex is an important mechanism that is involved in renovascular hypertension. Considering the proximity of the carotid bodies with the carotid baroreflex afferents emerging from the carotid sinuses, any investigation involving surgical removal of the carotid bodies should ensure that the baroreflex afferents are left intact.

Despite these open questions, the pioneering study by Melo et al. [12] set the framework for advancing knowledge toward understanding the role of carotid bodies in processing of renovascular hypertension at the cNTS level. Whether the carotid bodies are essential or play a minor role in the development and maintenance of renovascular hypertension is a matter that warrants further investigation. Future clinical trials investigating how unilateral removal of the carotid bodies (because bilateral removal of the carotid bodies is not possible in humans) may affect blood pressure in renovascular hypertensive patients will be highly relevant. A possible positive outcome from those clinical trials may add to the current antihypertensive therapy arsenal.

Funding VAB is funded by the Conselho Nacional de Desenvolvimento Científico e Tecnológico - CNPq (VAB: 472133/2013-6; 304772/2014-3; and 429767/2016-1) and Coordenação de Aperfeiçoamento de Pessoal de Nível Superior - Capes (VAB: Capes/STINT 1499/2014).

\section{Compliance with ethical standards}

Conflict of interest The author declares that he has no conflict of interest.
Publisher's note: Springer Nature remains neutral with regard to jurisdictional claims in published maps and institutional affiliations.

\section{References}

1. Garovic VD, Textor SC. Renovascular hypertension and ischemic nephropathy. Circulation. 2005;112:1362-74.

2. Goldblatt H, Lynch J, Hanzal RF, Summerville WW. Studies on experimental hypertension: I. The production of persistent elevation of systolic blood pressure by means of renal ischemia. J Exp Med. 1934;59:347-79.

3. DeForrest JM, Knappenberger RC, Antonaccio MJ, Ferrone RA, Creekmore JS. Angiotensin II is a necessary component for the development of hypertension in the two kidney, one clip rat. Am J Cardiol. 1982;49:1515-7.

4. Braga VA, Medeiros IA, Ribeiro TP, Franca-Silva MS, Botelho-Ono MS, Guimaraes DD. Angiotensin-II-induced reactive oxygen species along the SFO-PVN-RVLM pathway: implications in neurogenic hypertension. Braz J Med Biol Res. 2011;44:871-6.

5. Young CN, Davisson RL. Angiotensin-II, the brain, and hypertension: an update. Hypertension. 2015;66:920-6.

6. Biancardi VC, Son SJ, Ahmadi S, Filosa JA, Stern JE. Circulating angiotensin II gains access to the hypothalamus and brain stem during hypertension via breakdown of the blood-brain barrier. Hypertension. 2014;63:572-9.

7. Cutsforth-Gregory JK, Benarroch EE. Nucleus of the solitary tract, medullary reflexes, and clinical implications. Neurology. 2017;88:1187-96.

8. Kumar P, Prabhakar NR. Peripheral chemoreceptors: function and plasticity of the carotid body. Compr Physiol. 2012;2:141-219.

9. Pijacka W, Moraes DJ, Ratcliffe LE, Nightingale AK, Hart EC, da Silva MP, et al. Purinergic receptors in the carotid body as a new drug target for controlling hypertension. Nat Med. 2016;22: 1151-9.

10. Narkiewicz K, Ratcliffe LE, Hart EC, Briant LJ, Chrostowska M, Wolf $\mathrm{J}$, et al. Unilateral carotid body resection in resistant hypertension: a safety and feasibility trial. JACC Basic Transl Sci. 2016;1:313-24.

11. Nogueira V, Brito-Alves J, Fontes D, Oliveira L, Lucca W, Tourneur Y, et al. Carotid body removal normalizes arterial blood pressure and respiratory frequency in offspring of proteinrestricted mothers. Hypertens Res. 2018;41:1000-12.

12. Melo MR, Gasparini S, Speretta GF, Silva EF, Pedrino GR, Menani JV, et al. Importance of the commissural nucleus of the solitary tract in renovascular hypertension. Hypertens Res. 2019;42. https://doi.org/10.1038/s41440-018-0190-6

13. Nunes FC, Braga VA. Chronic angiotensin II infusion modulates angiotensin II type I receptor expression in the subfornical organ and the rostral ventrolateral medulla in hypertensive rats. J Renin Angiotensin Aldosterone Syst. 2011;12:440-5. 